\title{
Initial Experience of Pembrolizumab Therapy in Japanese Patients With Metastatic Urothelial Cancer
}

\author{
SHOTARO YASUOKA ${ }^{1,2}$, TAKESHI YUASA ${ }^{1}$, NORIKO NISHIMURA ${ }^{3}$, \\ MASAHIRO OGAWA ${ }^{1}$, YOSHINOBU KOMAI ${ }^{1}$, NOBORU NUMAO ${ }^{1}$, \\ SHINYA YAMAMOTO ${ }^{1}$, YUKIHIRO KONDO ${ }^{2}$ and JUNJI YONESE ${ }^{1}$ \\ ${ }^{1}$ Department of Urology, Cancer Institute Hospital, Japanese Foundation for Cancer Research, Tokyo, Japan; \\ ${ }^{2}$ Department of Urology, Nippon Medical School Hospital, Tokyo, Japan; \\ ${ }^{3}$ Department of Hematology, Cancer Institute Hospital, Japanese Foundation for Cancer Research, Tokyo, Japan
}

\begin{abstract}
Background/Aim: Pembrolizumab was approved as second-line treatment for patients with metastatic urothelial cancer $(U C)$ in Japan. We performed a retrospective pilot study to assess the potency of pembrolizumab treatment in Japan. Patients and Methods: The medical records of 40 consecutive Japanese patients with metastatic UC who started pembrolizumab between January and October 2018 were reviewed and statistically analyzed to clarify the efficacy and safety of the drug. Results: The objective response rate, median progressionfree survival period, and median overall survival period were 20.6\%, 4.1 months and 10.0 months, respectively. Multivariate analysis indicated the presence of liver metastasis, worse performance status $(\geq 2)$, and higher $C$-reactive protein as factors predictive of shorter OS. Conclusion: We demonstrated for the first time, a comparable efficacy and safety profile of pembrolizumab for Japanese patients with metastatic UC, as in the KEYNOTE045 study. The results indicate the features of pembrolizumab therapy in the current Japanese clinical practice.
\end{abstract}

Cisplatin-based chemotherapy continues to be the mainstay of systemic therapy for patients with metastatic urothelial cancer (UC) (1). In 1985, Sternberg et al. reported the remarkable results of the multi-agent chemotherapy regimen, known as MVAC (methotrexate, vinblastine, adriamycin, cisplatin) $(1,2)$. Thereafter, a clinical trial comparing a gemcitabine-plus-cisplatin regimen (GC) with MVAC

Correspondence to: Shotaro Yasuoka, MD, Department of Urology, Cancer Institute Hospital, Japanese Foundation for Cancer Research, 3-8-31 Ariake, Koto-ku, Tokyo 135-8550, Japan. Tel: +81 335200111, Fax: +81 335200141, e-mail: shotaro.yasuoka@jfcr.or.jp

Key Words: Pembrolizumab, urothelial carcinoma, immune related adverse events, immune checkpoint inhibitor. revealed that the GC regimen had similar treatment efficacy to that of MVAC, with lower toxicity $(2,3)$. Therefore, the GC regimen is currently widely used as the standard firstline treatment. However, no standard second-line treatment had been established until the acceptance of pembrolizumab.

Pembrolizumab, a highly selective humanized monoclonal IgG4 $\mathrm{K}$ isotype antibody against programmed death 1 (PD-1) protein, was approved for use in Japan on December 25, 2017, as a second-line treatment for platinum-based chemotherapy-resistant patients with metastatic UC, due to the better efficacy and safety profile reported in the international randomized phase III trial, KEYNOTE-045 (2, $4,5)$. However, information regarding the efficacy and adverse events of this treatment is limited to the results of clinical trials, with no evidence from actual clinical practice $(4,5)$. The present study retrospectively examined the therapeutic outcomes and safety profiles of pembrolizumab in Japanese patients with metastatic UC after platinum-based chemotherapy in a real-world clinical practice.

\section{Patients and Methods}

The medical records of patients with previous platinum-based chemotherapy for the treatment of metastatic UC who received pembrolizumab as second or later line therapy at our institution between January and October 2018 were retrospectively reviewed. This study received the approval of the institutional review board of the Cancer Institute Hospital, Japanese Foundation for Cancer Research (2012-1008). Before the initial treatment, all patients gave their written informed consent for pembrolizumab treatment.

Treatment and follow-up examination. Pembrolizumab (200 mg) was administered every 3 weeks, as previously described $(4,5)$. We recorded the patients' medical history, including physical examination findings, Eastern Cooperative Oncology Group performance status (ECOG-PS), laboratory findings and chest radiography data before starting treatment and during pembrolizumab therapy, as assessed based on the attending physician's discretion. As the cut-off value of laboratory findings, upper limit of normal range (ULN) or lower limit 
Table I. Patient characteristics $(n=40)$.

Age (years)

Male/Female

Pathological grade: 1 or $2 / 3$

Primary tumor site

Upper urinary tract (renal pelvis, ureter)

Radical nephroureterectomy

Bladder

Radical cystectomy

ECOG-PS: 0/1/2/3

Number of prior regimens: $1 / 2 / 3$

Metastatic sites

Lung/Liver/Lymph node/Bone

Hemoglobin (mg/dl): $>10 /<10$

CRP (C-reactive protein: $0.5 \mathrm{mg} / \mathrm{dl}$ ): $<3 />3$

LDH (Lactate dehydrogenase): $<$ ULN $/ \geq$ ULN

NLR (neutrophil-lymphocyte ratio)

Time from previous chemotherapy (months): $\geq 3 /<3$

Bellumunt risks: 0, 1, 2, 3

Previous chemotherapy regimen

Gemcitabine/cisplatin

Gemcitabine/carboplatin

Gemcitabine/cisplatin/etoposide

Gemcitabine/cisplatin/paclitaxel

Methotrexate/vinblastine/adriamycin/cisplatin
69 (range $=44-83$ years $)$

$32(80 \%) / 8(20 \%)$

$5(12.5 \%) / 35(87.5 \%)$

$19(47.5 \%)$

$18 / 19(95 \%)$

$21(52.5 \%)$

$10 / 21(47.5 \%)$

$20(50 \%) / 14(35 \%) / 5(12.5 \%) / 1(2.5 \%)$

$15(37.5 \%) / 21(52.5 \%) / 4(10 \%)$

$18(45 \%) / 6(15 \%) / 35(87.5 \%) / 8(20 \%)$

$26(65 \%) / 14(35 \%)$

$17(42.5 \%) / 23(57.5 \%)$

$31 /(77.5 \%) / 9(22.5 \%)$

$11 / 29$

$10(25 \%) / 30(75 \%)$

$15(37.5 \%), 12(30 \%), 11(27.5 \%), 2(5 \%)$

$20(50 \%)$

$17(42.5 \%)$

$15(37.5 \%)$

$14(35 \%)$

$3(7.5 \%)$

ECOG PS: Eastern Cooperative Oncology Group-performance status. Bellumunt risk assesses the number of risk factors present from among worse performance status $(>1)$, low hemoglobin $(<10 \mathrm{mg} / \mathrm{dl})$, presence of liver metastasis, and short interval from previous chemotherapy $(<3 \mathrm{months})$.

of normal range was employed except for C-reactive protein (CRP), hemoglobin $(\mathrm{Hb})$, and neutrophil lymphocyte ratio (NLR). According to the previously studies, $0.5 \mathrm{mg} / \mathrm{dl}, 10 \mathrm{~g} / \mathrm{dl}$, and 3 were employed as the cut-off value of CRP, $\mathrm{Hb}$, and NLR (6-8). The response to therapy was objectively evaluated by computed tomography every 2 or 3 months using the Response Evaluation Criteria in Solid Tumors (RECIST) guideline version 1.1 (9). Toxicity was assessed by the Common Terminology Criteria for Adverse Events (CTCAE) version $4.0(10)$.

Statistical analysis. Progression-free survival (PFS) and overall survival (OS) periods, defined as the period from initial administration of pembrolizumab until diagnosis of progressive disease and death from any cause, respectively, were assessed in all patients. Survival curves were estimated using the Kaplan-Meier method and compared using the log-rank test. All statistical analyses were performed using JMP software version 14.0 (SAS Institute Inc., Cary, NC, USA) and $p$-values $<0.05$ were considered significant.

\section{Results}

Patient characteristics. The study included 40 consecutive patients who were diagnosed with metastatic UC and commenced treatment with pembrolizumab after platinumbased chemotherapy at our hospital between January 2018 and October 2018. The characteristics of these patients are described in Table I. Twenty-one (52.5\%) and 19 patients (47.5\%) had bladder and upper urinary tract (UUT) UCs, respectively. Among these patients, 15 (37.5\%) and 25 patients $(62.5 \%)$ were administered pembrolizumab as the second and third or later line treatment, respectively.

Efficacy of pembrolizumab. The median follow-up period after pembrolizumab initiation was 5.3 months (range $=1.4$ 12.3 months). Thirty-four patients were evaluated for antitumor response and the objective response rate (ORR) was $20.6 \%$ (Figure 1A). Among the remaining six patients, two patients had no evaluable target lesion (small lymph node metastases), and four patients progressed clinically before the first imaging evaluation. In this study, all cases who demonstrated a good response with pembrolizumab therapy demonstrated tumor shrinkage within 3 months after pembrolizumab therapy, with no patient presenting initial tumor progression before shrinkage (pseudoprogression). In addition, the estimated median PFS period was 4.2 months (range=0.3-11.6 months) and the 3- and 6-month PFS rates were $61.5 \%$ and $43.7 \%$, respectively (Figure 1B). The median OS period was 10.0 months (range $=1.4-12.3$ months) and the estimated 3-, 6- and 12-month OS rates were 79.5\%, $58.9 \%$ and $49.1 \%$, respectively (Figure 1C).

Risk factors for short survival period. Next, we investigated variables that could predict a shorter OS period for patients with metastatic UC treated with pembrolizumab as second or 
A

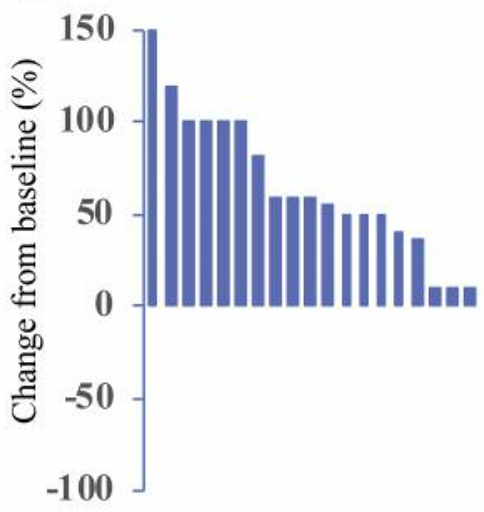

n

C

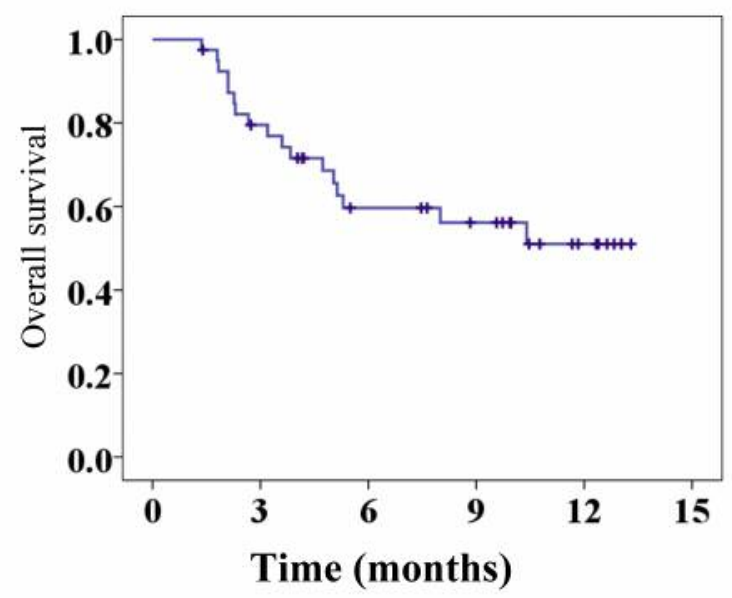

B

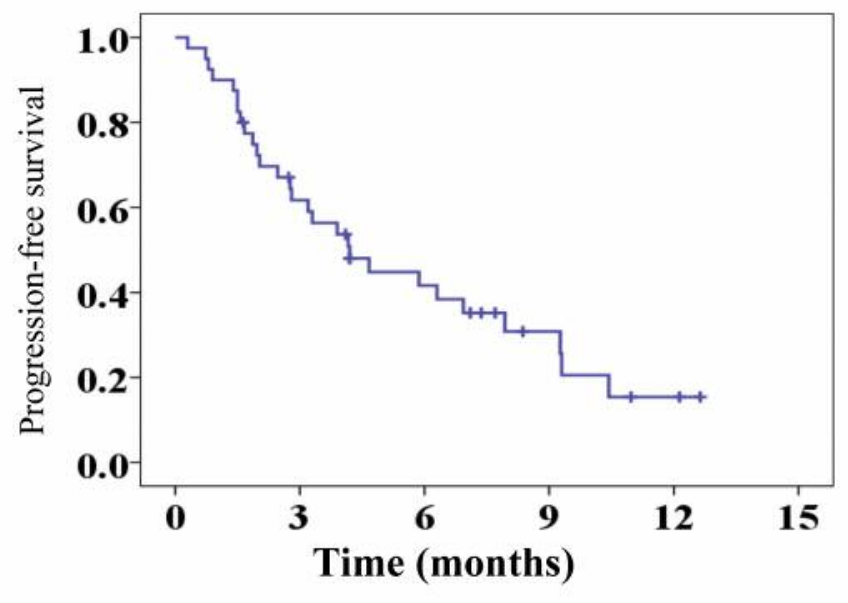

D

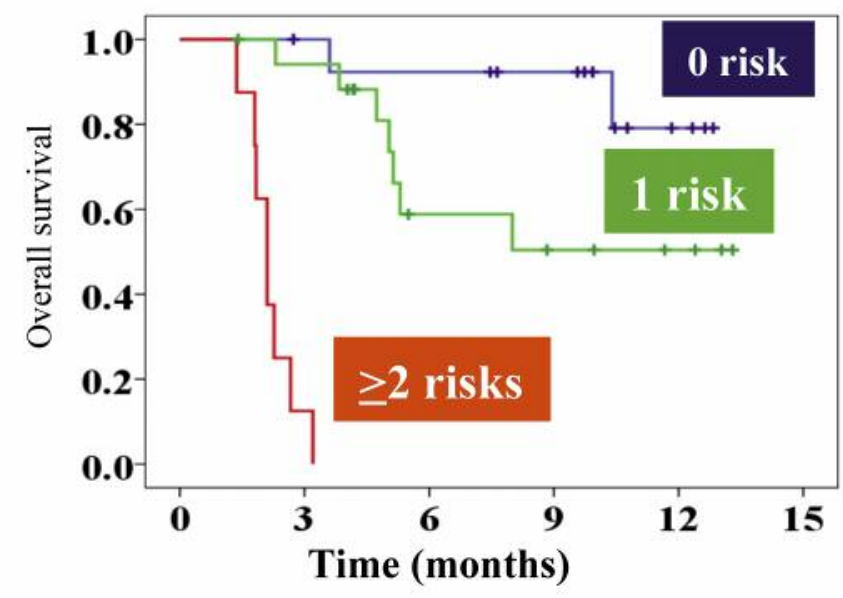

Figure 1. Efficacy of pembrolizumab as second- or more line treatment for Japanese patients with platinum-based chemotherapy-resistant metastatic urothelial cancer. Waterfall plots of the response to pembrolizumab $(A, n=34)$. Progression-free survival and overall survival curves $(B, C ; n=40)$. The prognostic model $(D)$. Our prognostic model, which included three risk factors, namely poor performance status $(\geq 2)$, presence of liver metastasis, and elevated C-reactive protein levels (>upper limit of normal range), stratified patients treated with pembrolizumab into three risk groups [favorable: 0 risk factors, $n=14$ (35\%); intermediate: 1 risk factor, $n=18$ (45\%); and poor: $\geq 2$ risk factors, $n=8(20 \%)$ ]. The prognostic model distinctly separated the overall survival curves of the three risk groups $(p<0.001)$.

later line therapy. On univariate analysis, worse PS $(\geq 2)$, liver metastasis, bone metastasis, visceral metastasis, high Creactive protein (CRP) levels ( $\geq \mathrm{ULN}$ : upper limit of normal range) and high lactate dehydrogenase levels ( $\geq$ ULN) were suggested as factors predictive of a shorter OS. On multivariate analysis, liver metastasis (hazard ratio $(\mathrm{HR})=31.1, \quad 95 \%$ confidence interval $(\mathrm{CI})=5.5-215.9$, $p<0.001)$, worse PS $(\geq 2) \quad(\mathrm{HR}=6.9,95 \% \mathrm{CI}=1.4-40.1$, $p=0.019)$, and higher CRP ( $>\mathrm{ULN})(\mathrm{HR}=6.0,95 \% \mathrm{CI}=1.8$ 27.8, $p=0.0028$ ) were reported as independent factors predictive of a shorter OS period (Table II).

Next, we established a prognostic model using these three risk factors to stratify patients treated with pembrolizumab into three risk groups: favorable [0 risk factors, $n=14(35 \%)$ ], intermediate [ 1 risk factor, $n=18(45 \%)]$, and poor $[\geq 2$ risk factors, $n=8(20 \%)]$ risk groups. The median OS periods of patients in the favorable, intermediate, and poor risk groups were not reached, 8.0 and 2.1 months, respectively (Figure 1D). The 6- and 12-month OS rates of patients in the favorable risk group were $92 \%$ and $79 \%$, respectively. In addition, the 6- and 12-month OS rates of patients in the intermediate risk group were $59 \%$ and $50 \%$, respectively. Conversely, the 3- and 6-month OS rates of patients in the poor risk group were $13 \%$ and $0 \%$, respectively. In this prognostic model, the OS curves of the three groups were distinctly separated from each other (Figure 1D, $p<0.001$ ). 
Table II. Univariate and multivariate analyses of prognostic factors for poor outcomes with pembrolizumab therapy.

\begin{tabular}{|c|c|c|c|c|c|}
\hline \multirow[t]{2}{*}{ Subgroup } & \multicolumn{2}{|c|}{ Univariate analysis } & \multicolumn{2}{|c|}{ Multivariate analysis } & \multirow[t]{2}{*}{ Reference category } \\
\hline & Hazard ratio $(95 \% \mathrm{CI})$ & $p$-Value & Hazard ratio $(95 \% \mathrm{CI})$ & $p$-Value & \\
\hline Gender & $0.82(2.94-0.29)$ & 0.741 & & & Female/Male \\
\hline Age & $1.31(3.42-0.47)$ & 0.593 & & & $<70 \mathrm{y} />70 \mathrm{y}$ \\
\hline ECOG PS & $19.72(97.19-4.97)$ & $<0.0001$ & $6.92(40.09-1.38)$ & 0.019 & $\geq \mathrm{PS} 2 / \mathrm{PS}=0.1$ \\
\hline Tumor site & $1.57(4.57-0.59)$ & 0.37 & & & LTUC/UTUC \\
\hline Surgical treatment & $0.61(1.69-0.23)$ & 0.33 & & & Yes/No \\
\hline Lung metastasis & $1.50(4.01-0.57)$ & 0.401 & & & Positive/Negative \\
\hline Liver metastasis & $18.91(68.84-5.40)$ & $<0.0001$ & $31.13(215.88-5.47)$ & $<0.0001$ & Positive/Negative \\
\hline Bone metastasis & $5.23(14.48-1.82)$ & 0.003 & & & Positive/Negative \\
\hline Visceral metastasis & $3.73(13.34-1.31)$ & 0.013 & & & Positive/Negative \\
\hline $\mathrm{Hb}$ & $1.71(4.50-0.64)$ & 0.28 & & & $>10 /<10$ \\
\hline LDH & $3.10(8.18-1.06)$ & 0.039 & & & $<250 />250$ \\
\hline CRP & $3.88(13.89-1.35)$ & 0.010 & $6.00(27.84-1.79)$ & 0.0028 & $<0.5 />0.5$ \\
\hline NLR & $1.09(3.45-0.40)$ & 0.86 & & & $<2 />2$ \\
\hline PLT count & $1.39(4.99-0.22)$ & 0.68 & & & $>40000 /<40000$ \\
\hline $\begin{array}{l}\text { TFPC (Time from } \\
\text { post-chemotherapy) }\end{array}$ & $1.53(6.66-0.50)$ & 0.48 & & & $<3$ months/ $>3$ months \\
\hline
\end{tabular}

ECOG PS: Eastern Cooperative Oncology Group-performance status; LTUC: lower tract urothelial carcinoma; UTUC: upper tract urothelial carcinoma; Hb: hemoglobin, LDH: lactate dehydrogenase; CRP: C-reactive protein; PLT: plate NLR: neutrophil-lymphocyte ratio.

Adverse events. Eighteen patients (45\%) experienced adverse events (AE), including four (10\%) with severe AE (grade 3 or more), as described in Table III. The most common AE was rash $(n=8,20 \%)$, although none of them were of a severe grade. Four patients $(10 \%)$ discontinued pembrolizumab therapy due to AEs.

Among the patients who experienced severe AEs, one patient died due to grade 5 hemophagocytic syndrome. This patient had polymyalgia rheumatica (PMR), which was being treated with $5 \mathrm{mg}$ prednisolone. We decided to start pembrolizumab therapy with adequate informed consent after a total of 11 cycles of platinum-based chemotherapy. However, after the first administration of pembrolizumab, he complained of fatigue and joint stiffness, which was diagnosed as exacerbation of PMR. The dose of prednisolone was increased from $5 \mathrm{mg}$ to $15 \mathrm{mg}$, resulting in subjective improvement of his symptoms. However, two months after pembrolizumab was first administered, his platelet count decreased $\left(19,000 / \mathrm{mm}^{3}\right)$ without any other abnormal signs ( $\mathrm{PS}=0$ ). He was emergently admitted to our hospital and referred to the department of hematology where he underwent bone marrow biopsy and was diagnosed with hemophagocytic syndrome. We immediately commenced steroid pulse therapy $(1,000 \mathrm{mg}$ for 3 days $)$ followed by intravenous immune globulin, cyclosporine administration and plasma exchange, with appropriate antibiotic and transfusion support. Unfortunately, the hemophagocytic syndrome did not improve and he died of multiple organ dysfunction 13 days after admission. In this case, the possibility of immune-related $\mathrm{AE}$ due to pembrolizumab was considered.
Table III. Pembrolizumab treatment-related adverse events $(n=40)$.

\begin{tabular}{lll}
\hline & Grade 1-2 & Grade 3-5 \\
\hline $\begin{array}{l}\text { Number of patients with AEs } \\
\text { Types of AEs }\end{array}$ & $15(37.5 \%)$ & $4(10 \%)$ \\
Rash & $8(20 \%)$ & 0 \\
Hypothyroidism & $4(10 \%)$ & 0 \\
Adrenal insufficiency & $1(2.5 \%)$ & $2(5 \%)$ \\
Hepatic dysfunction & $1(2.5 \%)$ & $1(2.5 \%)$ \\
Interstitial pneumonia & $2(5 \%)$ & 0 \\
Diarrhea & $1(2.5 \%)$ & 0 \\
Dysgeusia & $1(2.5 \%)$ & 0 \\
Hemophagocytic syndrome & 0 & $1(2.5 \%)$ \\
\hline
\end{tabular}

AEs: Adverse events.

\section{Discussion}

In this study, we reported our initial experience with pembrolizumab in Japanese patients with metastatic UC patients. The ORR was $20.6 \%$ and the estimated median PFS and OS were 4.2 months and 10.0 months, respectively (Figure 1A, B and C). Three, 6- and 12-month OS rates were $79.5 \%, 58.9 \%$ and $49.1 \%$, respectively. All these results are comparable with those reported in the international phase III trial of pembrolizumab for patients with metastatic UC, i.e. the KEYNOTE-045 trial, which only included patients with relatively good PS (<ECOG PS1). On the other hand, since our study is a real-world clinical practice retrospective study, 
it included 6 patients (15\%) with relatively poor PS (ECOG PS2 or 3). Our study demonstrated that caution is needed when adopting pembrolizumab therapy for a poor PS patient population.

All cases who demonstrated good response with pembrolizumab therapy experienced tumor shrinkage within 3 months after pembrolizumab induction. With immune checkpoint inhibitor therapy, the phenomenon of pseudoprogression, in which the lesions apparently temporarily worsen followed by subsequent tumor shrinkage, has been reported (11). In our study, although we did not encounter any cases of pseudoprogression, in real world clinical practice there might be a slight possibility of subsequent improvement in UC patients who do not initially respond well to therapy within 3 months.

In this study, presence of liver metastasis, worse PS ( $\geq 2)$, and higher CRP levels $(>\mathrm{ULN})$ were extracted as independent predictors of a poor prognosis. Previously, Bellumunt et al. also reported that liver metastasis, poor PS $(>1)$, and hemoglobin less than $10 \mathrm{mg} / \mathrm{dl}$ were poor prognostic factors in metastatic UC patients after platinum-based chemotherapy (9). Additionally, short period since the last dose of prior chemotherapy $(<3$ months) was also extracted as a poor prognostic factor in their study, and patients were stratified according to these four factors in the KEYNOTE-045 trial (4, 12). However, elevation of CRP levels, which is a representative acute phase reactant that is widely used to evaluate systemic inflammation, is known to be a poor predictor of advanced UC, as described previously (6). Therefore, the risk factors of poor prognosis that were extracted in this study, namely presence of liver metastasis, worse PS and higher CRP levels, might be advanced UC-related poor prognostic factors rather than pembrolizumab therapy-related risk factors.

Regarding adverse events, $10 \%$ of the patients experienced grade 3 or more immune-related AEs (irAEs), and $10 \%$ of patients discontinued treatment due to the AEs. These results were not significantly different compared to the pivotal KEYNOTE-045 trial (4). In our study, one case presented with grade 5 irAE. This case had a pre-existing auto-immune disease (polymyalgia rheumatica) and was on steroid therapy (prednisolone $5 \mathrm{mg}$ ). Clinical trials, including the KEYNOTE045 study, usually exclude patients with pre-existing autoimmune diseases. Menzies et al. reported the efficacy and safety of anti-PD-1 therapy for melanoma patients with a preexisting auto-immune disease $(\mathrm{n}=52)$. Among them, ORR was $33 \%$ and 20 patients (38\%) had a flare up of auto-immune disease requiring immunosuppression, although only two patients (4\%) discontinued treatment due to disease flare up (13). Although anti-PD-1 therapy has been reported to induce immune toxicities relatively frequently in patients with preexisting auto-immune diseases, these were often reported to be mild and easily managed (13-17). However, our experience of incurable irAE in a patient with pre-existing auto-immune disease highlights the need for careful patient selection and appropriate informed consent in this patient population. Further, hematologic disorders, including hemophagocytic syndrome caused by immune checkpoint inhibitors, is considered to be a relatively uncommon irAE (18-20). Although many of these cases were reported to be manageable with standard treatment algorithms, we must remember that hematologic disorders are potentially lethal irAEs.

We conducted this retrospective pilot study to clarify the efficacy and safety of pembrolizumab therapy in Japanese patients. Major limitations of our study are its retrospective design and the small cohort size. However, no large, multiinstitutional, and prospective or retrospective studies for pembrolizumab therapy have been previously published from Japan. Our findings describe the features of pembrolizumab therapy for metastatic UC patients in current clinical practice in Japan.

\section{Conclusion}

In this study, we demonstrated, for the first time, a comparable efficacy and safety profile of pembrolizumab therapy for Japanese patients with metastatic UC in real-world clinical practice as in the KEYNOTE-045 trial. Liver metastasis, worse PS and higher CRP levels were extracted as poor prognostic factors, and the prognostic model that we established using these factors showed distinctly separate OS curves for these factors. Patients with pre-existing auto-immune disease might experience irAEs and/or autoimmune exacerbations more frequently and of greater severity, suggesting the need for obtaining adequate informed consent from them before therapy, as well as the need to monitor patients closely in collaboration with other clinical departments.

\section{Conflicts of Interest}

T. Yuasa received remuneration for lectures from Astellas (Tokyo, Japan), Sanofi Japan (Tokyo, Japan), Pfizer Japan (Tokyo, Japan), Novartis Pharma Japan (Tokyo, Japan), Ono Pharma (Osaka, Japan), Bristol-Myers Squibb Japan (Tokyo, Japan), MSD Japan (Tokyo, Japan), Janssen Japan (Tokyo, Japan), and Daiichi-Sankyo (Tokyo, Japan). The other Authors have no conflicts of interest to declare.

\section{Authors' Contributions}

Study Conception and Design: SY and TY, Manuscript writing: SY and TY, Statistical analysis: SY, TY, SY and JY, Coordination of the team and final corrections: JY, Patient's management: TY, NN, MO, YK, NN, SY and JY. All Authors read and approved the final manuscript.

\section{Acknowledgements}

This work was partly supported by the Smoking Research Foundation and JSPS KAKENHI Grant Number 16K11035 (T.Y.) 


\section{References}

1 Sternberg CN, Yagoda A, Scher HI, Watson RC, Ahmed T, Weiselberg LR, Geller N, Hollander PS, Herr HW, Sogani PC, et al:: Preliminary result of M-VAC (methotrexate, vinblastine, doxorubicin, and cisplatin) for transitional cell carcinoma of the urothelium. J Urol 133: 403-407, 1985. PMID: 4038749.

2 Yuasa T, Urakami S and Yonese J: Recent advances in medical therapy for metastatic urothelial cancer. Int J Clin Onc 23: 599607, 2018. PMID: 29556919. DOI: 10.1007/s10147-018-1260-0

3 Von der Maase H, Sengelov L, Roberts JT, Dogliotti L, Oliver T, Moore MJ, Bodrogi I, Albers P, Knuth A, Lippert CM, Kerbrat P, Sanchez Rovira P, Wersall P, Cleall SP, Roychowdhury DF, Tomlin I, Visseren-Grul CM and Conte PF: Gemcitabine and cisplatin versus methotrexate, vinblastine, doxorubicin, and cisplatin in advanced or metastatic bladder cancer: results of a large, randomized, multinational, multicenter, phase III study. J Clin Oncol 18: 3068-3077, 2000. PMID: 11001674. DOI: $10.1200 / J C O .2000 .18 .17 .3068$

4 Bellmunt J, de Wit R, Vaughn DJ, Fradet Y, Lee JL, Fong L, Vogelzang NJ, Climet MA, Petrylak DP, Choueiri TK, Necchi A, Gerritsen W, Gurney H, Quinn DI, Culine S, Sternberg CN, Mai Y, Poehlein CH, Perini RF and Bajorin DF: Pembrolizumab as second-line therapy for advanced urothelial carcinoma. N Engl J Med 376: 1015-1026, 2017. PMID: 28212060. DOI: 10.1056/NEJMoa1613683

5 Vaughn DJ, Bellmunt J, Fradet Y, Lee JL, Fong L, Vogelzang NJ, Climent MA, Petrylak DP, Choueiri TK,Necchi A, Gerritsen W, Gurney H, Quinn DI, Culine S, Sternberg CN, Mai Y, Li H, Perini RF, Bajorin DF and Ronald de Wit: Health-Related Quality-of-Life Analysis From KEYNOTE-045: A Phase III study of pembrolizumab versus chemotherapy for previously treated advanced urothelial cancer. J Clin Oncol 36: 1579-1587, 2018. PMID: 29590008. DOI: 10.1200/JCO.2017.76.9562

6 Tanaka N, Kikuchi E, Shirotake S, Kanao K, Matsumoto K, Kobayashi H, Miyazaki Y, Ide H, Obata J, Hoshino K, Hayakawa N, Ito Y, Kosaka T, Kodaira K, Oyama M, Miyajima A, Momma T, Nakagawa K, Ueno M and Oya M: The predictive value of C-reactive protein for prognosis in patients with upper urothelial carcinoma treated with radical nephroureterectomy: a multi-institutional study. Eur Urol 65: 227-234, 2014. PMID: 23219372. DOI: $10.1016 /$ j.eururo.2012.11.050

7 Bellmunt J, Choueiri TK, Fougeray R, Schutz FAB, Salhi Y, Winquist E, Culine S, Maase HVD, Vaughn DJ and Rosenberg JE: Prognostic factors in patients with advanced transitional cell carcinoma of the urothelial tract experiencing treatment failure with platinum-containing regimens. J Clin Oncol 28: 1850-1855, 2010. PMID: 20231682. DOI: 10.1200/JCO.2009.25.4599

8 Tan YG, Eu EWC, Huang HH and Lau WKO: High neutrophilto-lymphocyte ratio predicts worse overall survival in patients with advanced/metastatic urothelial bladder cancer. Int J Urol 25: 232-238, 2010. PMID: 29094397. DOI:10.1111/iju.13480

9 Eisenhauer E.A, Therasse P, Bogaerts J, Schwartz LH, Sargent D, Ford R, Dancey J, Arbuck S, Gwyther S, Mooney M, Rubinstein L, Shankar L, Dodd L, Kaplan R, Lancombe D and Verweij J: New response evaluation criteria in solid tumours: Revised RECIST guideline (version 1.1). Eur J Cancer 45: 228247, 2009. PMID: 19097774. DOI: 10.1016/j.ejca.2008.10.026

10 National Cancer Institute. Common Terminology Criteria for Adverse Events (CTCAE) version 4.0. http://evs.nci.nih.
gov/ftp1/CTCAE/CTCAE_4.03_2010-06-14_Quick Reference_ 8.5x11.pdf. Accessed 9 February 2018.

11 Chiou VL and Burotto M: Pseudoprogression and immunerelated response in solid tumors. J Clin Oncol 33: 3541-3543, 2015. PMID: 26261262. DOI: 10.1200/JCO.2015.61.6870

12 Sonpavde G, Pond R G, Fougeray R Choueiri TK, Qu AQ, Vaughn DJ, Niegisch G, Albers P, James ND, Wong YN, Ko YJ, Sridhar SS, Galsky MD, Petrylak DP, Vaishampayan UN, Khan A, Volgelzang NJ, Beer TM, Stadler WM, O'Donnell PH, Sternberg CN, Rosenberg JE and Bellmunt J: Time from prior chemotherapy enhances prognostic risk grouping in the secondline setting of advanced urothelial carcinoma: a retrospective analysis of pooled, prospective phase 2 trials. Eur Urol 63: 717723, 2018. PMID: 23206856. DOI: 10.1016/j.eururo.2012. 11.042

13 Menzies MA, Johnson BD, Ramanujam S, Atkinson VG, Wong ANM, Park JJ, Mcquade JL, Shoushtari AN, Tsai KK, Eroglu Z, Klein O, Hassel JC, Sosman JA, Guminski A, Sullivan RJ, Ribas A, Carlino MS, Davies MA, Sandhu SK and Long GV: Anti-PD1 therapy in patients with advanced melanoma and preexisting autoimmune disorders or major toxicity with ipilimumab. Ann Oncol 28: 368-376, 2017. PMID: 27687304. DOI: 10.1093/ annonc/mdw443

14 Donia M, Pedersen M and Svane MI: Cancer immunotherapy in patients with preexisting autoimmune disorders. Semin Immuno 39: 333-337, 2017. PMID: 27730287. DOI: 10.1007/s00281016-0595-8

15 Johnson BD, Sullivan JR, Ott AP, Carlino MS, Khushalani NI, Ye F, Guminski A, Puzanov I, Lawrence DP, Buchbinder EI, Mudigonda T, Spencer K, Bender C, Lee J, Kaufman HL, Menzies AM, Hassel JC, Mehnert JM, Sosman JA, Long GV and Clark JI: Ipilimumab therapy in patients with advanced melanoma and preexisting autoimmune disorders. JAMA Oncol 2: 234-240, 2016. PMID: 26633184. DOI: 10.1001/jamaoncol. 2015.4368

16 Kyi C, Carvajal DR, Wolchok DJ and Postow MA: Ipilimumab in patients with melanoma and autoimmune disease. J Immune Ther Cancer 2: 35, 2014. PMID: 25349698. DOI:10.1186/ s40425-014-0035-Z

17 El Osta B, Hu F, Sadek R, Chintalapally R and Tang SC: A meta-analysis of immune-related adverse events (irAE) of immune checkpoint inhibitors (ICI) from cancer clinical trials. Ann Oncol 27: vi359-vi378, 2016. PMID: 29065979. DOI: 10.1016/j.critrevonc.2017.09.002

18 Hantel A, Gabster B, Cheng JX, Golomb H and Gajewski TF: Severe hemophagocytic lymphohistiocytosis in a melanoma patient treated with ipilimumab + nivolumab. J Immunother Cancer 6: 73, 2018. PMID: 30012206. DOI: 10.1186/s40425018-0384-0

19 Sadaat M and Jang S: Hemophagocytic lymphohistiocytosis with immunotherapy: brief review and case report. J Immunother Cancer 6: 49, 2018. PMID: 29871698. DOI: $10.1186 / \mathrm{s} 40425-018$

20 Satzger I, Ivanyi P, Länger F, Kreipe HH, Schaper-Gerhardt K, Beutel G, Cornberg M and Gutzmer R: Treatment-related hemophagocytic lymphohistiocytosis secondary to checkpoint inhibition with nivolumab plus ipilimumab. Eur J Cancer 93: 150153, 2018. PMID: 29472154. DOI: 10.1016/j.ejca. 2018. 01.063

Received June 3, 2019

Revised June 10, 2019 Accepted June 14, 2019 